\title{
Ingeniería y arte en una relación escultórico-ambiental desde lo local hacia lo internacional.
}

\section{Engineering and art in a sculptural-environmental relationship from the local to the international.}

TIPO DE TRABAJO:

Comunicación.

PALABRAS CLAVE:

Escena, escultura, ingeniería, interactividad

KEY WORDS:

Scene, scuplture, engineering, interactivity

\section{RESUMEN.}

El trabajo presenta la relación entre la ingeniería robótica y las propuestas artísticas en un país como México. Se muestran las acaracterísticas del preoceso de creación que usa la tecnología emergente como soporte en comparación con otros paises a nivel internacional. Los logros obtenidos en el concurso ROBOCUP, colaborando la facultad de ingeniería de la UNAM y la facultad de artes y diseño de la misma institución. Como característica local trabajamos con recursos limitados sin qeu sea un defecto, nos enfrentamos con otros donde el concepto de tecnología tiene otras características. Es un análisis comparativo que muestra una estrategia de trabajo local tomando en cuenta los estandares internacionales, "Si la técnica es fundamentalmente (ontológicamente) un conjunto de decisiones que se manifistan necesarias y empíricamente en una práctica, entonces la filosoía de la técnica es en su base una filosofía de la acción, y más específicamente una filosofía de la acción intencional. " Con ésta idea la facultad de artes y diseño y la facultad de ingeniería de la UNAM nos hemos dado a la tarea de explorara las posibilidades plásticas de la robótica en un proyecto llamado "Desarrollo de técnicas de la robótica aplicadas a las artes escénicas y visuales" con el apoyo económico para la investigación de la misma institución y del cual se mostrarán avances materiales y el proceso conceptual que ha acompañado el proyecto.

\section{ABSTRACT.}

This paper presents the relationship between robotic engineering and artistic proposals in a country like Mexico. It shows the characteristics of the creative mind that uses emerging technology as a medium in comparison with other countries at an international level. The achievements obtained in the competition ROBOCUP, collaborating the engineering faculty of the UNAM and the faculty of arts and design of the same institution. As a local characteristic we work with limited resources without being a defect, we are faced with others where the concept of technology has other characteristics. It is a comparative analysis that shows a local work strategy taking into account the international standards, "If the technique is fundamentally (ontologically) a set of decisions that are necessary and empirical in a practice, then the philosophy of the technique is in its Base a philosophy of action, and more specifically a philosophy of intentional action. "With this idea the faculty of arts and design and the engineering faculty of the UNAM we have given to the task of exploring the plastic 
possibilities of robotics in A project called "Development of techniques of robotics applied to the scenic and visual arts" with the economic support for the research of the same institution and which will show material advances and the conceptual process that has accompanied the project.

\section{CONTENIDO.}

Es complicado desde una posición local y con la perspectiva de un país en vías de desarrollo (tercermundista dirían otros), hablar de tecnología cuando no ha generado propuestas particularmente sobresalientes o brillantes en lo internacional, como en su tiempo fue el caso del sistema tricromático de secuencias de campos para la televisión inventado por Guillermo González Camarena, mexicano, quien curiosamente es el hermano menor de Jorge González Camarena, un distinguido muralista que destaca por su trabajo de colores contrastantes y modelos indígenas. Quienes nacimos en los 60's del siglo anterior, recordamos los libros de educación básica que mostraban la imagen de "La Patria" obra encargada en 1962 al pintor por la Secretaría de Educación Pública; curiosa fue la relación entre hermanos, uno inclinado hacia el arte y el otro al desarrollo tecnológico. Ahora siglo XXI, integrados en este mundo sospechosamente globalizado, con las conveniencias que para algunos países encarna y de las que pocas veces salimos bien librados, nos vemos limitados en la producción de tecnologia y en gran medida nos conformamos con los parámetros establecidos desde el exterior. Seguimos siendo periferia tanto en el ambiente tecnológico como en el artístico, no tanto por nuestra incapacidad de generar propuestas en estos ámabitos, más por la falta de vías de investigación, financiación y estrategias de socialización. Esa es la realidad y tenemos la responsabilidad de trabajar con ella para hacernos notar, en la calle, en la ciudad, en los montes, en el mundo, desde nosotros.

Los grandes presupuestos son una ilusión y con ello lleva una desventaja, aunada a la falta generalizada de insumos locales sólo alimenta las ganancias de los verdaderamente grandes centros de desarrollo tecnológico. Lo que nos ocupa aquí es la generación de estrategias para pensar en la tecnología y en el arte en una dinámica de trabajo colaborativo desde nuestra realidad y posibilidades particulares; ante ello es obligado establecer un diálogo, en áreas vistas como separadas cuando realmente no es así, complementarías una de la otra. No es novedoso el uso de recursos tecnológicos en propuestas artísticas e incluso son ya una tendencia casi obligada en el contexto actual, donde el fluir de las obras de autores reconocidos ha tomado los nuevos medios de comunicación y los procesos de manufactura a través de la tecnología. Más allá de la materialidad y la tradición, encontramos que hacen falta estrategias de trabajo que incluyan a profesionales de diferentes áreas en proyectos complejos, con la propia diferencia profesional en términos de esquemas de pensamiento cualitativamente identificados, provenientes de ámbitos con una visión del otro a veces accesoria, otras inexistente. Para nuestra propuesta de trabajo la relación Arte-Ingeniería, se da a partir de la consideración que el artista realiza al observar desde fuera el recurso tecnológico, con la distancia del soporte frío que dará forma a sus propuestas, en contraparte, el ingeniero ve con la misma lejanía el trabajo del artista sin comprender su profundidad, la función social de su trabajo; situación esta que no tiene muchas variantes en el contexto internacional.

De inicio, encontrar los puntos que nos unen como creadores a requerido buscar definiciones que generen una visión compartida, distintiva, común, que de sentido al propio y particular quehacer profesional, la técnica para ambos modelos de profesión resuena como un principio unificador, encontramos que tanto artistas como ingenieros encuentran gran atractivo en la idea el saber hacer. Jaime Fisher aborda el problema de manera clara en la siguiente cita:

"Si la técnica es fundamentalmente (ontológicamente) un conjunto de decisiones que se manifiestan necesaria y empíricamente en una práctica, entonces la filosofía de la técnica es en su base una filosofía de la acción, y más específicamente una filosofía de acción intencional." ${ }^{1}$

Compartiendo eso, nos enfocamos en el detonante inicial, abordar lo escultórico como problema. Compartimos en concepto de forma y objeto plástico con más o menos palabras, la visón de uno y otro tienen diferencias que deben entrar en sintonía de ideas, llevar lo que se comprende como escultórico hacia la ingeniería puede ser complicado en ambas direcciones, es posible que el artista visual comprenda un poco más las implicaciones del término en el contexto actual, en el terreno conceptual es un problema a resolver en conjunto. En el proyecto de investigación que aquí se expone pretende mostrar las características y procesos que se han tenido que acordar y perfeccionar para llegar a los resultados materiales que también se expondrán.

La cuestión es supone romper las fronteras propias de la innovación, enriquecer el concepto de disciplina, tanto lo artístico como en lo no artístico, es importante comprender primero la pertinencia de la propuesta artística en un ambiente que por sí mismo ha dejado de respetar fronteras en pos de la rentabilidad comercial o de la experimentación honesta que siempre acompaña la actividad artística. Por su parte, las escuelas de arte están llegando tarde al trabajo de redefinición de las disciplinas artísticas, continúan los mismos talleres donde los alumnos desarrollarán las habilidades que los lleven a ejercer de manera mínimamente adecuada la

\footnotetext{
${ }^{1}$ Fisher, Jaime. El hombre y la técnica. Hacia una filosofía política de la ciencia y la tecnología. Ed. UNAM, 2010. México. Pp. 45.
} 
profesión de artista. En nuestras instituciones encontramos espacios divididos ya sea por materialidades, dimensiones, soportes o técnicas establecidas, algunos desde hace muy antiguos, con pocas posibilidades de transformarse, otros de más reciente creación que están pujando para ser reconocidos y tomados en cuenta. Nuevos talleres que en instituciones de gran calado como la UNAM corren el riesgo de subutilizar recursos en lugar de mejorar los proceso de investigación, varios elementos tecnológicos no se aprovechan adecuadamente en alguna facultad porque otra, que puede estar al lado, no sabe que la primera lo tiene y podría hacer acuerdos de uso colaborativo. Eso no solo mejoraría el uso de los recursos, también sumaría criterios de otros profesionales en formación o en activo a nuestras propuestas.

\section{Enfoque para conjunción de acuerdos}

Para el proyecto abordamos las artes escénicas como pretexto, roemoiendo tradiciones, consideramos su implicación multimediática, desde que Richard Wagner propuso su intención de crear el arte total, quién más que concebir a la tecnología como ajena a la actividad artística, concibe como responsabilidad indispensable el que el artista tome todo lo que la tecnología le provea. Ya Rosalind E. Krauss hacia 1977 se cuestionaba el papel del teatro en el juego de las artes, en su libro Paisajes de la escultura moderna dedica un capitulo completo al problema que nombra Ballets mecánicos: luz, movimiento y teatro, dice lo siguiente: ${ }^{2}$

En 1967 la inquietante sensación de que el teatro había invadido el terreno de la escultura recibió un ataque frontal. En esa época Michael Fried escribió:

...quiero hacer una afirmación que no tengo esperanzas de poder ni probar ni justificar, pero que, sin embargo, creo verdadera, a saber: que el teatro y la teatralidad están hoy en día en guerra no solo con la pintura moderna (o con la pintura y la escultura modernas), sino con el arte como tal y, en la medida en que las distintas artes pueden describirse como modernas, con la sensibilidad moderna como tal... El éxito e incluso la supervivencia de las artes depende cada vez más de su capacidad para vencer al teatro.

En apariencia una guerra declarada hacia las artes visuales y las fronteras del arte en su totalidad, legitima también el derecho de las artes visuales a tomar la escena como algo propio valiéndose del trabajo escénico que no necesariamente pertenece de manera exclusiva al teatro, problema del arte en todo su conjunto, Krauss continúa citando: ${ }^{3}$

De modo que teatralidad es una palabra paraguas bajo la cual podrían situarse el arte cinético y el lumínico, así como la escultura ambiental y la escultura tableu, junto con el más explicito arte de la performance, donde habría que incluir desde los happenings hasta los decorado escénicos construidos por Robert Rauschenberg para las coreografías de Marce Cunningham. Pero, puesto que entre los críticos de la escultura moderna teatralidad se ha convertido en un término polémico -peyorativo en el ensayo de Fried, elogioso para los adeptos de estas diversas modalidades artísticas- deberíamos tratar de desentrañar el concepto de teatralidad, porque es demasiado denso y confuso, y está lleno de contradicciones, así como de intenciones y motivos opuestos. La cuestión no es si ciertos artistas han querido apropiarse del espacio escénico o explotar el tiempo dramático proyectado por el movimiento real; la cuestión es porqué, y con que fines estéticos, han querido apropiarse de tales cosas o emplearlas.

Estás contradicciones se dan el ambiente de las artes, que entendemos como tradicionales, en el ambiente teatral se ha dado como entendido que los profesionales de otras áreas pueden e incluso deben participar de la escena, la individualidad cede a los objetivos de la propuesta conformada en dinámica colectiva. El artista plástico, en algunos casos, cree que al incluirse en equipos de trabajo escénico pierde su carácter de artista, salvo en aquellas propuestas que lo mantienen como el director único de la propuesta como es el caso de la instalación, el (o la) performance, el happening y sus variantes. Las fronteras en formatos o materialidades nunca han sido tan claras como ahora, el obstáculo a vencer no es a que área del arte pertenece qué o que propuesta invade las parcelas infranqueables de otros. Si la actitud artística es en esencia desobediente de las normas ¿por qué somos tan obedientes con los formatos que potencialmente tiene la propuesta plástica? Quizá el reto es más de ego que de fronteras. Superando los obstáculos de las fronteras disciplinarias, juntos, las Artes visuales y escénicas acuden al llamado de la tecnología y confluyen en materialidades, estrategias y formatos de consumo. La barrera que separa lo plástico de lo escénico se difumina y su catalogación lleva por senderos descriptivos que pocas veces se comprenden como auténtica categorización, algunos, es de mencionarse como cualidad, lo integran a la retórica discursiva de la propuesta con resultados afortunados. Para otros resulta un compromiso difícil por cumplir en un ambiente que al parecer ha dejado los formatos tradicionales para los anquilosados museos o espacios de representación tradicionales.

El equipo de investigación tuvo que afinar el término de nuevas tecnologías, en términos prácticos, antes de llegar a lo novedoso se necesita resolver el problema de la accesibilidad, lo nuevo no siempre es accesible material, ni económicamente. Existen tecnologías

\footnotetext{
${ }^{2}$ Krauss Rodalind E. Pasajes de la Escultura Moderna. España. Akal. 2010. 201-201p * (Fried, Michael. Art and Objethood: Essays and Reviews, Chicago and Londres, University of Chicago Press, 1998) citado por Krauss.

${ }^{3}$ Ibid., pp. 204-205
} 
nuevas cada vez, a las que algunos pueden acceder en su uso, comprensión y manipulación, en nuestra posición el acceso debe pasar por un proceso distinto y en ocasiones caro, a veces por la misma condición, inviable. Las nuevas tecnologías se trasladan al terreno de las tecnologías accesibles, muchas de las cuales en realidad son improvisación tecnológica cuando recurrimos a las opciones open source o al copyleft y apresurar algunas soluciones, el objetivo principal por supuesto es generar la mayor cantidad de soluciones al interior del proyecto en la misma dinámica de la creación de tecnología, debe tomarse en cuenta que hay mucha gente trabajando en premisas similares a las nuestras.

En la primera vertiente del trabajo conjunto del robot de servicio Justina hay algunos elementos comparativos con otros equipos que compiten en la misma categoría, los cuales se exponen a continuación:

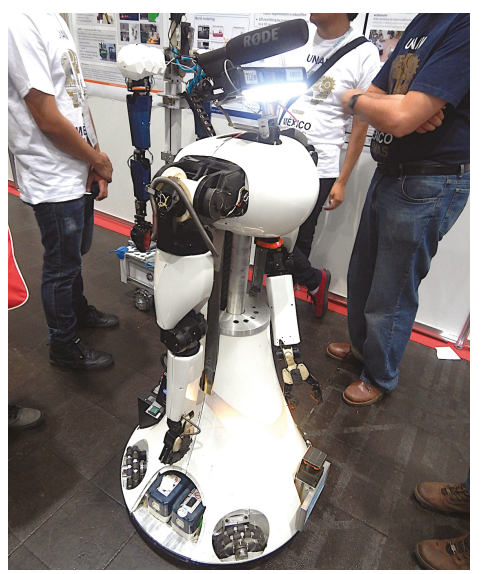

Foto tomada en la competencia de robótica "RoboCup 2016" en Leipzig, Alemania. Muestra al robot del equipo Tech United de la Universidad de Tecnología de Eindhoven.

La diferencia de esta propuesta con el trabajo realizado en el laboratorio de la UNAM radica en que buscamos que Justina tenga un aspecto amable para interactuar con los humanos, la interfaz mecatrónica que se incorpora en el robot mexicano mejora la interacción humano-robot. Con el robot Justina no solo se busca que cumpla satisfactoriamente las pruebas que la competencia exige, también se trabaja en el aspecto físico con la elaboración de carcasas utilizando Manufactura Aditiva Modelado por Deposición de Material Fundido (FDM por sus siglas en inglés), con diseños y colores que hacen referencia a la identidad de la Universidad y de México. Más allá de la identificación institucional, se busca establecer algunos parámetros iniciales para el diseño.

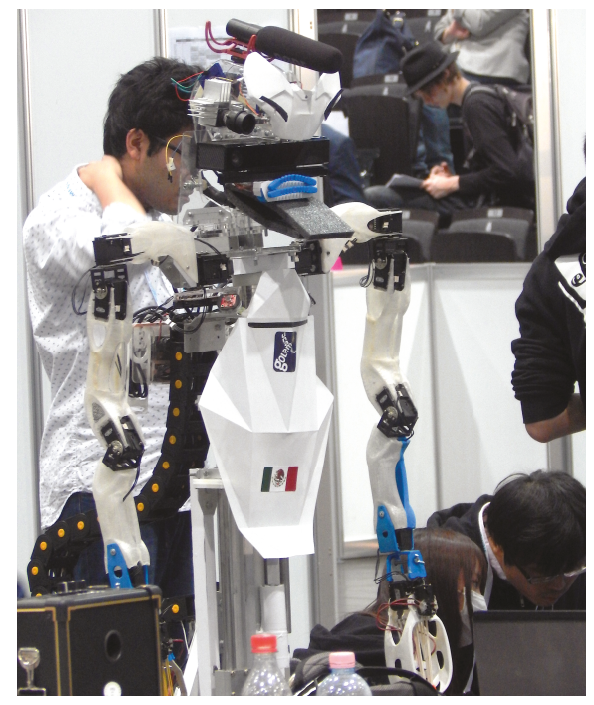

Foto tomada en la competencia de robótica "RoboCup 2016" en Leipzig, Alemania.

Muestra al robot del equipo Golem, de la UNAM. Grupo de investigación y desarrollo tecnológico del

Departamento de Ciencias de la Computación del Instituto de Investigaciones en Matemáticas Aplicadas y en sistemas (IIMAS) de la (UNAM). 
En la funcionalidad de la propuesta el robot debería cumplir con las metas establecidas, dando cuenta del trabajo conjunto realizado entre arte e ingeniería, encuentra un nuevo paradigma llevar el centro de interés hacia el problema visual y a la interacción emotiva, en el sentido funcional y estético.
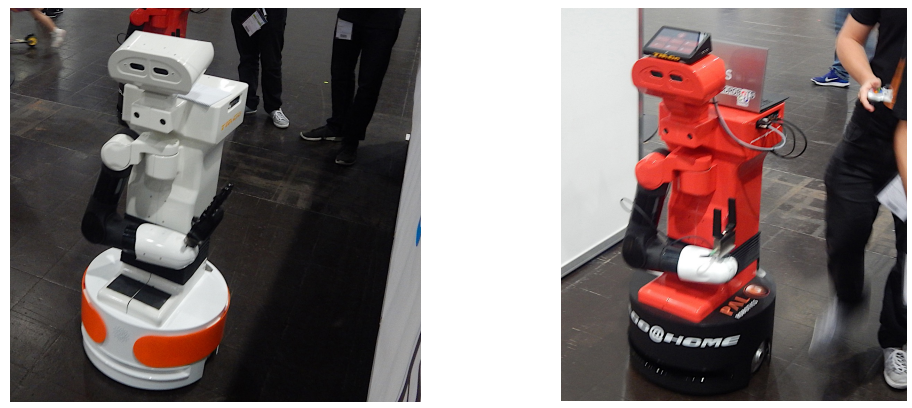

Foto tomada en la competencia de robótica "RoboCup 2016" en Leipzig, Alemania. Muestra a los robots de servicio comerciales de la empresa PAL robotics. No han competido en la RoboCup, están en venta para investigación y compañía. Utiliza un solo brazo manipuladora diferencia del robot Justina que incorpora dos.

Éste y el ejemplo siguiente muestran dos opciones comerciales de diseño, destaca su apariencia grata por los procesos industriales, los que evidentemente, al tratarse de una empresa dedicada a la comercialización, están mejor elaborados y pulcros en su presentación.

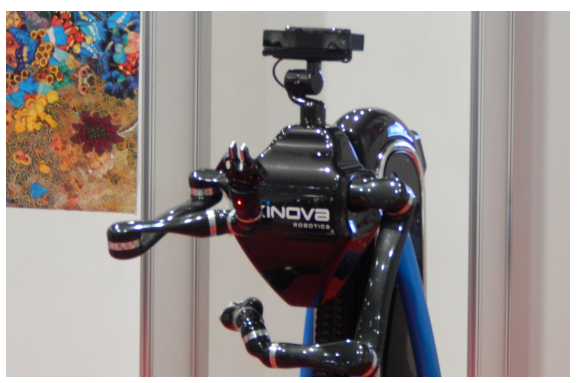

Foto tomada en la competencia de robótica "RoboCup 2016" en Leipzig, Alemania. Muestra al robot de servicio de la empresa Kinova Robotics. No ha competido en la RoboCup, se encontraba haciendo una demostración de venta para los equipos que competían. Los brazos manipuladores son lo mejor del robot, combinan ligereza y precisión -es su mejor característica-. Al ser un robot que asista a los humanos, no desarrollaron la parte de interacción humano-robot como el trabajo que se realiza en el laboratorio de bio- robótica de la UNAM.

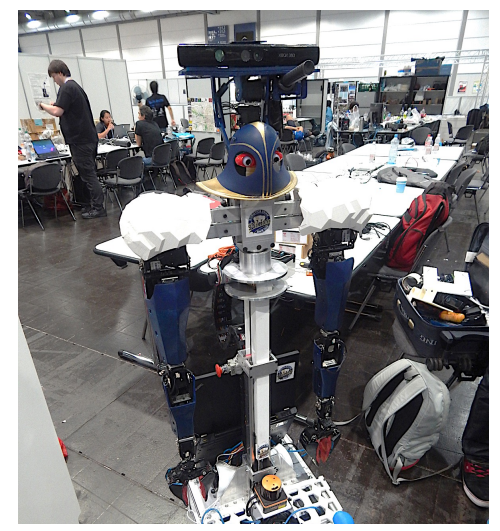

Foto tomada en la competencia de robótica “RoboCup 2016" en Leipzig, Versión inicial de Justina para el encuentro, actualmente en proceso de diseño. 
El elemento de rediseño central fue la cabeza, o lo que se podría interpretar como tal, la ubicación asociativa de elementos con el cuerpo humano no necesariamente cumple funciones similares, tratándose como recipiente de los órganos principales de percepción hacia el mundo, es también el elemento de empatía más apto para la interacción humana.
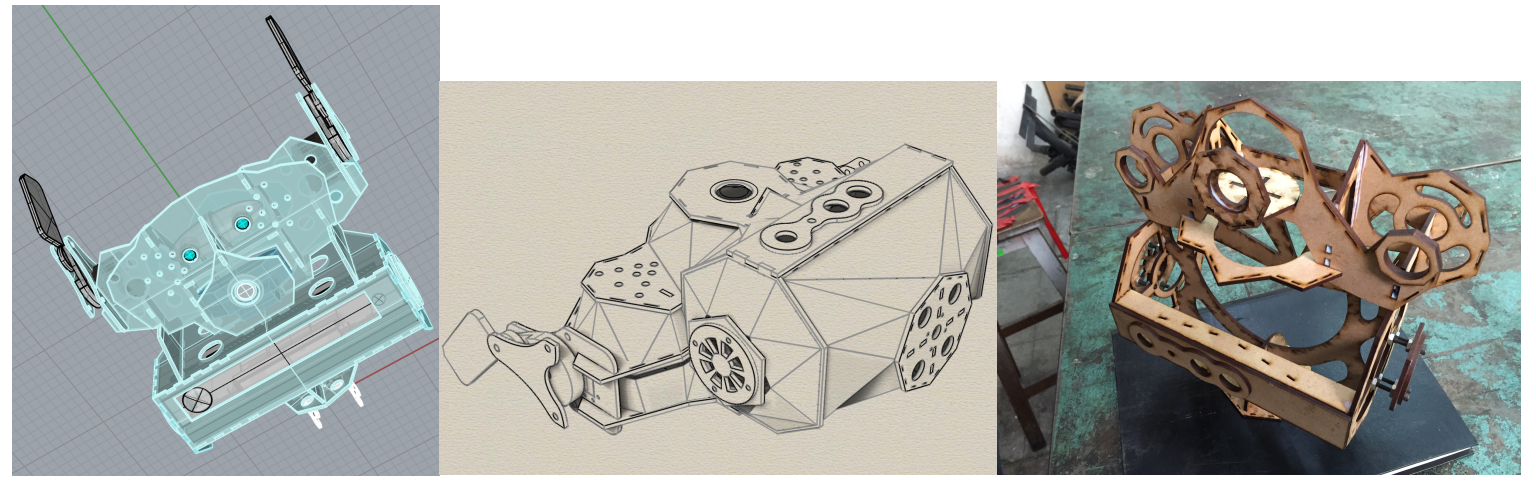

Modelado digital y primer corte laser en MDF segundo en acrílico y modelo actual.
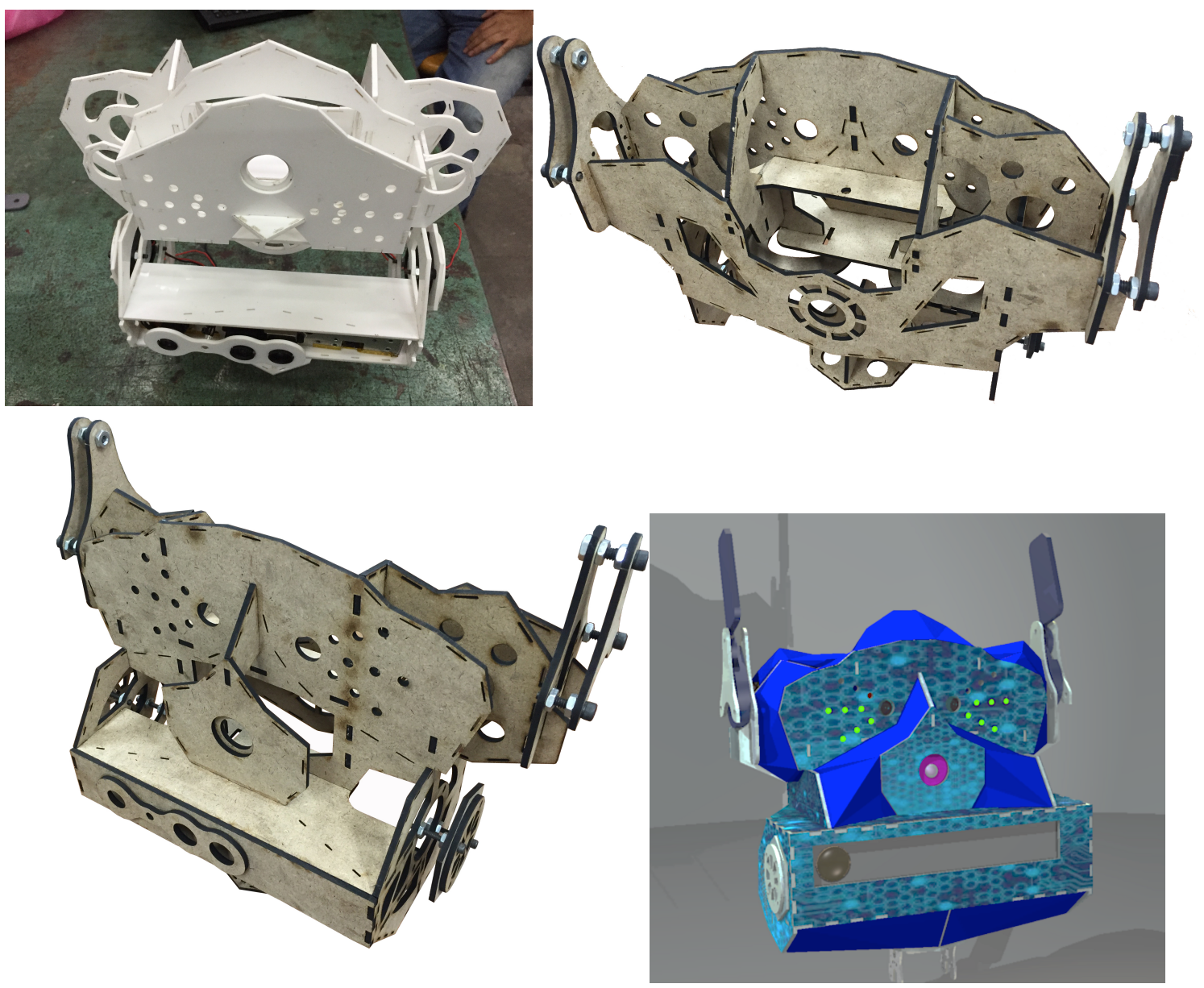

Modelo actual en corte MDF y modelado digital con acabados. 
La segunda vertiente del trabajo conjunto se está concretando a partir de una propuesta escénica dónde un robot recorre un circuito preestablecido en un espacio transitable, las características de interacción abarcan desde la implementación de sensores, imagen generativa en video y la programación de escenas consecutivas. El robot cubre la totalidad del espacio con un proyector que a su vez es manipulado para dirigir la imagen a diferentes partes del espacio, todo son elementos programados y manufacturados bajo un diseño artístico claro, propio, vinculado con las propuestas de ingeniería sobre las posibilidades tecnológicas.

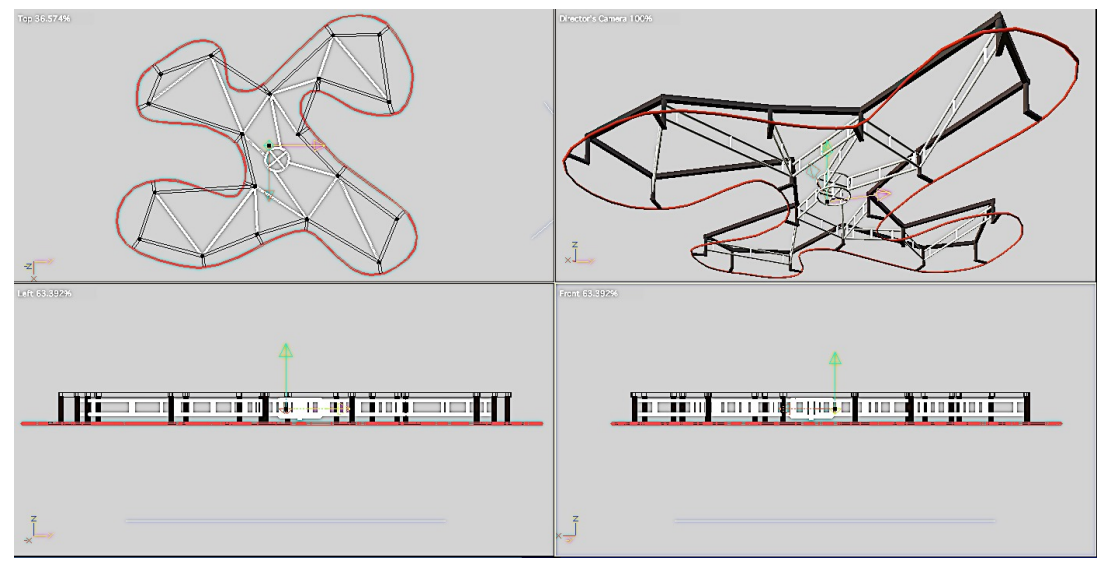

Plano del sistema de tránsito del robot.

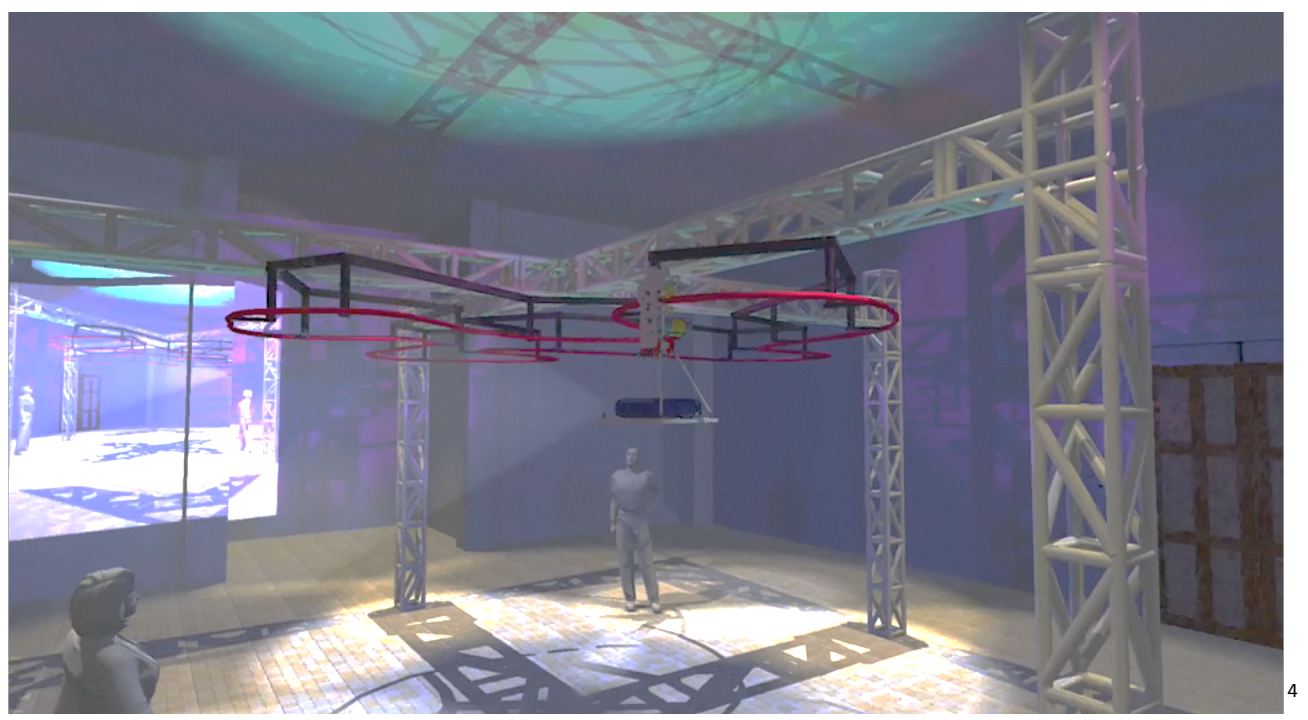

Fotograma de animación parte del diseño original.

\footnotetext{
${ }^{4}$ Participantes del proyecto de investigación: Arce González Héctor Rodrigo, Marentes Cruz Juan Manuel, Pano Sanjuan Manuel Alejandro, Savage Carmona Jesús, Villaseñor Bello José Francisco. Este trabajo es apoyado por PAPIIT-DGAPA UNAM (RG100915), como parte del proyecto "Desarrollo de técnicas de la robótica aplicadas a las artes escénicas y visuales". Se le agradece a la DGAPAUNAM el apoyo recibido.
} 

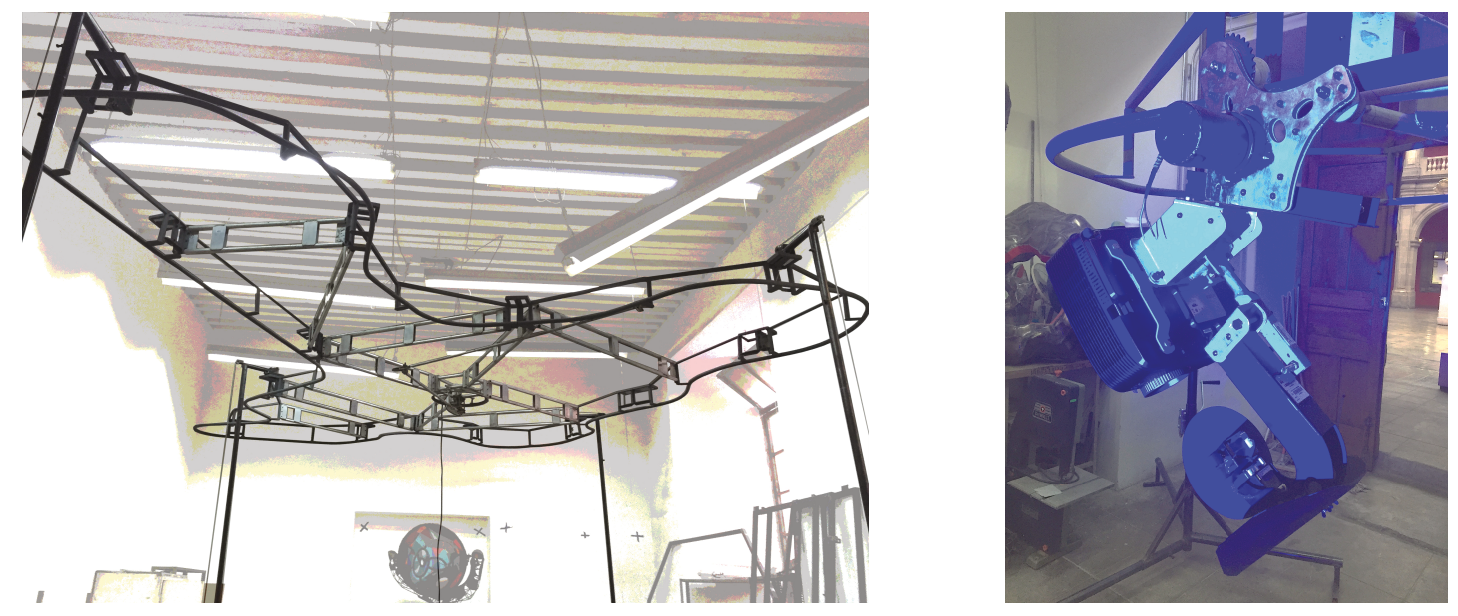

Montaje físico de estructura principal y el robot en primeras pruebas.

El sistema de programación se basa en el protocolo DMX, cuenta con interfaces de control establecidos para el ambiente del espectáculo y las artes escénicas en general, lo que ha servido para retomar algunos aditamentos en el mercado que son funcionales y accesibles. Se han presentado obstáculos de inaccesibilidad local aún con un mercado abierto para las compras en línea, oportunidad está para la improvisación y búsqueda conjunta de soluciones. En la primera vertiente del trabajo es evidente la desventaja en la competencia por los elementos comparativos internacionales que se tienen, en la segunda, al ser una propuesta más libre obliga a buscar símiles internacionales con quien establecer un diálogo.

El diálogo como método de disertación ha permitido alcanzar a materializar la obra, haciendo más productiva la empresa, discutiendo, flexibilizando, construyendo en el andar; queda pendiente el proceso de socialización tanto en el ambiente de las artes plásticas y en el de la ingeniería, sin olvidar que los alcances escénicos invitan a la interacción. La propuesta final se vislumbra con la participación de performers escénicos, bailarines, actores con roles específicos y espectadores en inmersión del espacio propuesto. Encontramos áreas de oportunidad en el diseño y manipulación de aditamentos robóticos, en la iluminación escénica, la integración de ambos perfiles profesionales permite encontrar vertientes tanto para la formación como para el diseño, en su uso, en la generación de nuevos instrumentos de iluminación manipulada robóticamente. Cabría ampliar la participación de otros profesionales interesados en el trabajo multidisciplinario, las conclusiones materiales, más allá de ser el resultado más espectacular, sirve de base para que cada uno de los integrantes amplíe la visión parcelaria de lo que se entiende como disciplina profesional, tarea que, en proyectos como el presente, deje de ser visto como novedad y se convierta en norma de la investigación de cualquier área. 